\title{
Malignant Otitis Externa and Stroke
}

\author{
Marta Catarino Manso ${ }^{1}$, Simão C. Rodeia ${ }^{1}$, Sofia Rodrigues ${ }^{1}$, Pedro Cavilhas ${ }^{2}$, Raquel Domingos ${ }^{1}$ \\ ${ }^{1}$ Department of Internal Medicine, Hospital de Egas Moniz, Lisboa, Portugal \\ ${ }^{2}$ Department of Otorhinolaryngology, Hospital de Egas Moniz, Lisboa, Portugal
}

Received: 14/01/2016

Accepted: 09/03/2016

Published: 14/04/2016

How to cite this article: Manso CM, Rodeia SC, Rodrigues S, Cavilhas P, Domingos R. Malignant otitis externa and stroke. EJCRIM

2016;3:doi:10.12890/2016_000387

Conflicts of Interests: The Authors declare that there are no competing interests.

This article is licensed under a Commons Attribution Non-Commercial 4.0 License

\section{ABSTRACT}

Malignant otitis externa (MOE) is an aggressive but benign entity which evolves into skull base osteomyelitis. An 81-year-old female patient was admitted for left hemiparesis and homonymous hemianopia. She complained of headache radiating to the right cervical area. A recent history of recurrent otitis media was present. Head and neck imaging showed an ischemic infarction (right temporo-occipital) and a parapharyngeal soft tissue mass originating in an external and medial ear infection. Culture samples revealed Pseudomonas aeruginosa infection leading to the diagnosis of Malignant otitis externa (MOE). Parenteral antibacterial therapy and hyperbaric oxygen therapy resulted in improvement.

\section{LEARNING POINTS}

- Malignant otitis externa is an aggressive and life-threatening disease which must be identified early and treated promptly for therapeutic success.

- Although starting as an outer ear infection (typically caused by Pseudomonas aeruginosa) local spread can involve noble head and neck structures leading to variable signs and symptoms.

- Modern imaging techniques can help define structure involvement (MRI) and may help identify disease activity and prognosis (nuclear medicine).

\section{KEYWORDS}

Malignant otitis externa, stroke, skull base osteomyelitis, internal carotid vasculitis.

\section{INTRODUCTION}

Malignant otitis externa (MOE) is an infection with a benign aetiology but aggressive behaviour that starts in the outer ear and evolves as a skull base osteomyelitis. In cases of external otitis refractory to therapy, especially in patients with diabetes, it is important to consider this entity, as early diagnosis and aggressive treatment improve patient prognosis.

\section{CASE REPORT}

An 81-year-old women was admitted to the emergency department with left body muscle weakness and visual disturbances evident during the previous 2 days. In the past month, she had complained of occipital headache radiating to the right cervical region.

The patient had a history of arterial hypertension and type 2 diabetes with multiple vascular complications. There was also a history of 
chronic otitis media, with exacerbation episodes during the previous year. She was being treated with metformin, glibenclamide, isosorbide mononitrate, sertraline and trazodone.

Clinical examination revealed preserved superior cognitive functions, no language alterations, left homonymous hemianopia, hypotonicity of the left palate, left deviation at tongue protrusion, flaccid left brachiocrural hemiparesis and left hemihypoesthesia, globally diminished deep tendon reflexes and indifferent left plantar response (amputation below the right knee). She was afebrile, mildly hypertensive and not in respiratory distress. No other significant alterations were noted.

Blood analysis showed poor glycaemic control (HbA1c 9\%) and elevated inflammatory markers (13,600 leukocytes/ $\mu$ l, neutrophilia, C-reactive protein $10.5 \mathrm{mg} / \mathrm{dl}$ (normal $<0.5 \mathrm{mg} / \mathrm{dl}$ ), sedimentation rate $69 \mathrm{~mm} / 1^{\text {st }}$ hour).

Head computerized tomography (CT) showed non-recent ischaemic lacunar lesions in the right pons and parietal-occipital region. In the cervical region, a mass $(3 \times 3 \times 3.7 \mathrm{~cm})$ was identified in the right lateral parapharyngeal space, demonstrating contrast enhancement and necrotic areas. Endoscopic observation revealed normal nasopharyngeal mucosa, albeit bulging towards the lumen. Right otoscopy revealed a thick exudate, a small polypoid mass of granulomatous appearance in the external auditory conduit posterior wall and tympanic perforation. Culture of pharyngeal aspirate and outer ear exudate revealed Pseudomonas aeruginosa with sensitivity to piperacillin/ tazobactam, gentamycin and ciprofloxacine. A transnasal sphenoidal bone biopsy was performed confirming the presence of $P$. aeruginosa. Brain magnetic resonance imaging (MRI) showed an extensive infiltrative lesion in the right-sided structures of the skull base, involving the pharyngeal mucosal space, prevertebral space, bony constituents of the skull base, and middle and external ear, with an abscess at its centre. The initiating condition seemed to be severe otitis externa and media, with external auditory canal stricture, osteitis of several bony structures of the right hemiskull, and right internal carotid vasculitis, with subocclusive stenosis near the entrance of the carotid canal (yellow arrowheads in Fig. 1). A recent right cortico-subcortical temporal-occipital-parietal ischaemic lesion was noted on the brain parenchyma (red arrowhead, right panel in Fig. 1). The vasculitis of the internal carotid associated with the inflammatory MOE process was possibly the cause of the ischaemic stroke noted on MRI.

Gallium-67 scintigraphy showed hyperfixation in a location compatible with the outer ear and the right skull base (Fig. 2).

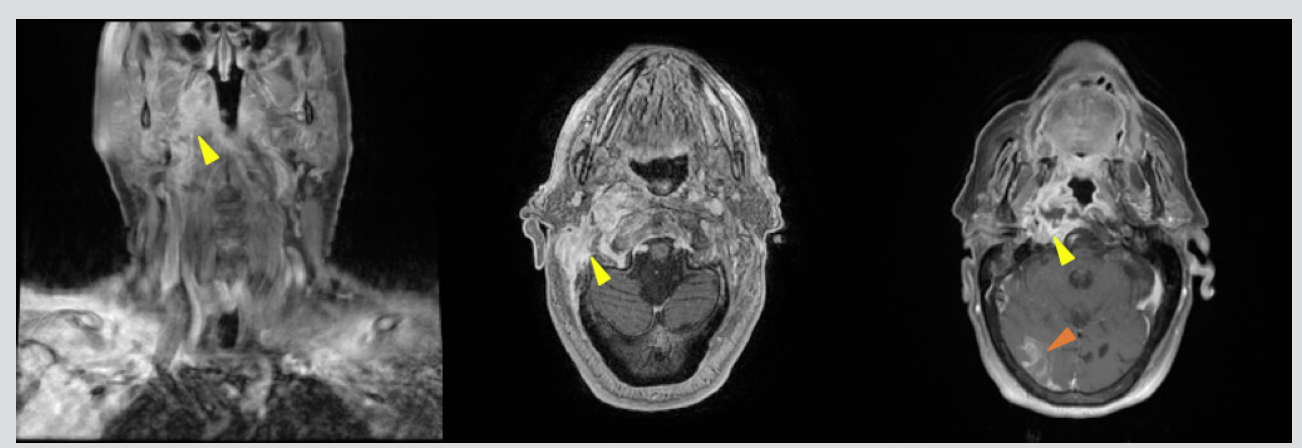

Figure 1: Magnetic resonance imaging showing an extensive soft tissue mass with extension to bony structures of skull base and an ischaemic lesion of the right occipital lobe.

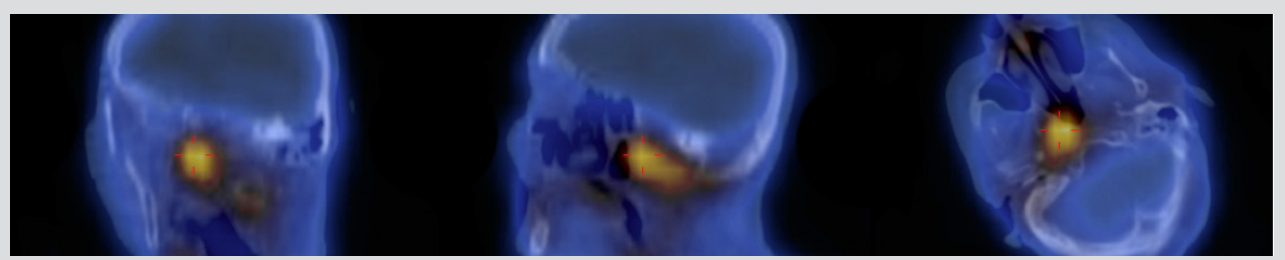

Figure 2: Gallium-67 scintigraphy showing hyperfixation in a location compatible with the outer ear and the right skull base. 
Empirical antibacterial therapy was started and adjusted according to the results of sensitivity tests. The patient completed a 6-week course of piperacillin/tazobactam and gentamicin, together with oxygen hyperbaric therapy. Clinical improvement was noted: there was remission of the neck pain but focal deficits persisted. Re-evaluation CT showed a reduction in the inflammatory process with partial remineralisation of bony structures. The patient was discharge under ciprofloxacin $750 \mathrm{mg}$ twice daily. However, 1 month after discharge a sudden deterioration in mental status culminated in her death (cause not evaluated).

\section{DISCUSSION}

MOE starts in the external auditory canal and progresses to skull base osteomyelitis. Although benign, it is invasive and can be fatal if diagnosis and treatment are delayed ${ }^{[1]}$. The major predisposing factor is diabetes mellitus, present in $90-100 \%$ of patients, the majority of whom have poor metabolic control ${ }^{[1,2]}$. The causative microorganism is P. aeruginosa in up to $98 \%$ of cases. The clinical presentation is variable but pain is frequently present (otalgia, headache and cervicalgia).

As the infection spreads locally, the facial (VII), glossopharyngeal (IX), vagus (X), accessory (XI) and rarely, the hypoglossal (XII) cranial nerves are involved at their emergence from the skull base. Such involvement is a marker of advanced disease but has no correlation with prognosis $^{[3]}$.

Central nervous system involvement varies from confusional states to severe complications such as meningitis, dural venous sinus thrombosis or brain abscess ${ }^{[4]}$.

There are no clinical findings or tests for MOE. The diagnosis relies on clinical suspicion, laboratory tests and imaging. In the present case, the most characteristic finding (not pathognomonic) was observed: a granulomatous lesion in the floor of the external auditory canal.

Brain computerized tomography (CT) results correlate with disease activity, establish the extent of bone destruction, and allow follow-up ${ }^{[2]}$. Magnetic resonance allows finer assessment of the inflammatory process but is not suitable for follow-up ${ }^{[3]}$. Nuclear medicine examination results are unable to evaluate the anatomical extent of disease but show correlation with disease activity ${ }^{[3]}$.

Treatment of MOE relies on antibacterial therapy with anti-pseudomonal activity for 6-8 weeks. The main choice is ciprofloxacin due to bone penetration and oral availability ${ }^{[2]}$. Some advocate oxygen hyperbaric therapy as an adjuvant therapy, although its efficacy has not yet been thoroughly demonstrated ${ }^{[5]}$.

Recurrence rates are high (15-20\%/year) and combined clinical, analytical and imaging (CT and gallium scintigraphy) follow-up is recommended.

Since adequate therapy has become available, mortality has significantly reduced from $50 \%$ to $0-15 \%{ }^{[3]}$.

\section{REFERENCES}

1. Guevara N, et al. Initial management of necrotizing external otitis: errors to avoid. Eur Ann Otorhinolaryngol Head Neck Dis 2013;130:115-121.

2. Grandis JR, Branstetter BF, Yu VL. The changing face of malignant (necrotising) external otitis: clinical, radiological, and anatomic correlations. Lancet Infect Dis 2004;4:34-39.

3. Carfrae MJ, Kesser BW. Malignant otitis externa. Otolaryngol Clin North Am 2008;41:537-549.

4. Bellia E, Galea R, Vassallo MA. Acute confusion and sudden deterioration in an elderly patient with necrotizing otitis externa. Eur Geriatr Med 2012;3:242-243.

5. Philips JS, Jones SE. Hyperbaric oxygen as an adjuvant treatment for malignant otitis externa. Cochrane Database Syst Rev 2013;5:CD004617. 